PROCEEDINGS OF THE

AMERICAN MATHEMATICAL SOCIETY

Volume 127, Number 1, January 1999, Pages 167-172

S 0002-9939(99)04748-6

\title{
REMARKS ON BLOW-UP BEHAVIOR FOR A NONLINEAR DIFFUSION EQUATION WITH NEUMANN BOUNDARY CONDITIONS
}

\author{
KENG DENG AND MINGXI XU \\ (Communicated by Jeffrey B. Rauch)
}

\begin{abstract}
We establish the blow-up rate for the solution of a nonlinear diffusion equation $\left(u^{m}\right)_{t}=u_{x x}, 0<x<1, t>0$, subject to Neumann boundary conditions $u_{x}(0, t)=0, u_{x}(1, t)=u^{\alpha}(1, t)$.
\end{abstract}

\section{INTRODUCTION}

In this paper, we consider the initial-boundary value problem

$$
\begin{array}{lll}
\left(u^{m}\right)_{t}=u_{x x}, & 0<x<1, & t>0, \\
u_{x}(0, t)=0, u_{x}(1, t)=u^{\alpha}(1, t), & & t>0, \\
u(x, 0)=u_{0}(x) \geq \delta>0, & 0 \leq x \leq 1 . &
\end{array}
$$

Here $0<m, \alpha<\infty, u_{0} \in C^{2+\nu}([0,1])$ for some $0<\nu<1$, and $u_{0}^{\prime}(0)=0$, $u_{0}^{\prime}(1)=u_{0}^{\alpha}(1)$.

Such a problem was previously studied in [5], wherein the following results were established.

Case $1.0<m<1$. If $\alpha \leq m$, every solution of problem (1.1) exists globally while the solution blows up in a finite time $T$ for $\alpha>m$. Moreover, if $m<\alpha \leq 1$, blow-up occurs on the whole interval $[0,1]$, and there exist two positive constants $C_{1}$ and $C_{2}$ such that

$$
C_{1}(T-t)^{1 /(m-\alpha)} \leq u(x, t) \leq C_{2}(T-t)^{1 /(m-\alpha)} \quad \text { for }(x, t) \in[0,1] \times[0, T) .
$$

If $m<1<\alpha$, blow-up occurs only at $x=1$.

Case 2. $m>1$. If $2 \alpha<m+1$, problem (1.1) possesses a global solution, whereas for $2 \alpha>m+1$, every solution must blow up in a finite time $T$. Furthermore, if $\alpha>m$, blow-up occurs only at $x=1$. And for $2 \alpha>m+1$, under an additional condition, the solution $u$ satisfies

$$
C_{\epsilon}(T-t)^{1 /(m+1-2 \alpha-\epsilon)} \leq u(1, t) \leq C(T-t)^{1 /(m+1-2 \alpha)} \quad \text { for } t \in[0, T)
$$

with $\epsilon \in(0,1)$ and $C_{\epsilon}, C>0$. The condition is as follows.

Received by the editors May 6, 1997.

1991 Mathematics Subject Classification. Primary 35B40, 35K20, 35K55.

(C)1999 American Mathematical Society 
(H) For some $\lambda>0, u_{0}(x) \in C^{\infty}([0,1])$ is a positive solution of the problem

$$
\begin{aligned}
& f^{\prime \prime}(x)=\lambda m f^{m+\alpha-1}(x) \quad 0<x<1, \\
& f^{\prime}(0)=0, f^{\prime}(1)=f^{\alpha}(1) .
\end{aligned}
$$

Later, it was shown in [8] that the critical case $2 \alpha=m+1$ belongs to the global existence part. When $m=1$, the nonlinear diffusion equation reduces to the heat equation, and (1.1) has been extensively studied (see [4], [6] and the literature cited therein).

Several questions remain open: What is the blow-up rate for the case $m<1<\alpha$ ? Compared to the result for $m=1$ in [4], can the lower bound in (1.3) be improved? And where does blow-up occur for the case $(m+1) / 2<\alpha \leq m$ ?

Our objective in this paper is to answer all the above questions. For convenience, letting $a(u)=(1 / m) u^{1-m}$ and $g(u)=u^{\alpha}$, in the sequel we shall mainly concentrate on the equivalent problem

$$
\begin{array}{lll}
u_{t}=a(u) u_{x x}, & 0<x<1, & 0<t<T, \\
u_{x}(0, t)=0, u_{x}(1, t)=g(u), & & 0<t<T, \\
u(x, 0)=u_{0}(x), & 0 \leq x \leq 1 . &
\end{array}
$$

\section{Blow-up RATE FOR THE CASE $m<1<\alpha$}

In this section we establish bounds on the blow-up rate for $m<1<\alpha$. We first present the lower bound.

Theorem 2.1. Let $m<1<\alpha$ and $u_{0}^{\prime}(x) \geq 0$ on $[0,1]$. Then there exists a positive constant $C_{3}$ such that

$$
u(1, t) \geq C_{3}(T-t)^{1 /(m+1-2 \alpha)} \quad \text { for } t \in[0, T) .
$$

Proof. Since $u_{0}^{\prime}(x) \geq 0$ for $0 \leq x \leq 1$, the maximum principle yields that $u_{x}(x, t) \geq$ 0 on $[0,1] \times[0, T)$. Inspired by [1], [3], we define a function $\Phi(x, t)=u_{x}(x, t)-$ $\varphi(x) g(u(x, t))$ in $(0,1) \times(0, T)$, where $\varphi(x)$ is a nonnegative function to be determined. Through a routine calculation, we obtain

$$
\begin{aligned}
\Phi_{t}= & a(u) \Phi_{x x}+a^{\prime}(u) u_{x} \Phi_{x} \\
& +\varphi(x)\left(a^{\prime}(u) g^{\prime}(u)+a(u) g^{\prime \prime}(u)\right) u_{x}^{2} \\
& +\varphi^{\prime}(x)\left(a^{\prime}(u) g(u)+2 a(u) g^{\prime}(u)\right) u_{x}+\varphi^{\prime \prime}(x) a(u) g(u) .
\end{aligned}
$$

Since $a^{\prime}(u) g^{\prime}(u)+a(u) g^{\prime \prime}(u)=(\alpha / m)(\alpha-m) u^{\alpha-m-1}>0$ and $a^{\prime}(u) g(u)+2 a(u) g^{\prime}(u)$ $=((2 \alpha-m+1) / m) u^{\alpha-m}>0$, if $\varphi(x)$ satisfies $\varphi^{\prime} \geq 0$ and $\varphi^{\prime \prime} \geq 0$ on $(0,1)$, then $\Phi$ cannot attain its negative minimum in $(0,1) \times(0, T)$.

Because $u_{0}^{\prime}(1)=u_{0}^{\alpha}(1), u_{0}^{\prime}(x)>0$ near $x=1$, we can construct $\varphi(x)$ as follows: $\varphi(x) \equiv 0$ for $0 \leq x \leq x_{0}$ with some $x_{0}<1$ and $\varphi(x)=\left(x-x_{0}\right)^{l} /\left(1-x_{0}\right)^{l}$ on $\left(x_{0}, 1\right]$, where $l(\geq 3)$ is chosen so large that $\varphi(x) \leq u_{0}^{\prime}(x) u_{0}^{-\alpha}(x)$ for $x_{0}<x \leq 1$. Then $\Phi(0, t)=\Phi(1, t)=0$, and $\Phi(x, 0) \geq 0$. By the maximum principle, we have $\Phi(x, t) \geq 0$, that is,

$$
u_{x}(x, t) \geq \varphi(x) g(u(x, t)) \quad \text { for }(x, t) \in[0,1] \times[0, T) .
$$

Moreover, $\Phi_{x}(1, t) \leq 0$, which means

$$
\begin{aligned}
u_{t}(1, t) & =a(u(1, t)) u_{x x}(1, t) \\
& \leq a(u(1, t)) g(u(1, t))\left(\varphi^{\prime}(1)+g^{\prime}(u(1, t))\right) \leq c_{0} u^{2 \alpha-m}(t) .
\end{aligned}
$$


Integrating (2.4) from $t$ to $T$ yields the asserted result.

We then give the upper bound, the derivation of which is in the spirit of [2].

Theorem 2.2. Let $m<1<\alpha$ and $u_{0}^{\prime}(x), u_{0}^{\prime \prime}(x) \geq 0$ on $[0,1]$. Then there exists a positive constant $C_{4}$ such that

$$
u(1, t) \leq C_{4}(T-t)^{1 /(m+1-2 \alpha)} \quad \text { for } t \in[0, T) .
$$

Proof. Since $u_{0}^{\prime \prime}(x) \geq 0$ for $0 \leq x \leq 1$, by the maximum principle one can see that $u_{x x}(x, t)$ and $u_{t}(x, t)$ are positive on $[0,1] \times[\tau, T)$ for any $\tau \in(0, T)$. Letting $\tau$ be close to $T$, consider $\Psi(x, t)=u_{t}(x, t)-\epsilon d(u(x, t)) u_{x}^{2-\sigma}(x, t)$ in $(1-T+\tau, 1)$ $\times(\tau, T)$, where $\epsilon$ is a positive constant, $(m+1) / \alpha<\sigma<(m+\alpha) / \alpha$, and $d(u)=$ $a(u) g^{\prime}(u) g^{\sigma-1}(u)$. By a tedious computation we find that

$$
\Psi_{t}=a(u) \Psi_{x x}+C(x, t) \Psi+\epsilon R(x, t) u_{x}^{4-\sigma}
$$

where

$$
\begin{aligned}
C(x, t)= & {\left[\frac{a^{\prime}(u)}{a(u)}+\epsilon(2-\sigma)(1-\sigma) \frac{d(u)}{a(u)} u_{x}^{-\sigma}\right] u_{t} } \\
& +\epsilon\left[(4-2 \sigma) d^{\prime}(u)-(1-\sigma) \frac{a^{\prime}(u) d(u)}{a(u)}\right] u_{x}^{2-\sigma} \\
& +\epsilon^{2}(2-\sigma)(1-\sigma) \frac{d^{2}(u)}{a(u)} u_{x}^{2-2 \sigma}
\end{aligned}
$$

and

$$
\begin{aligned}
R(x, t)= & a(u) d^{\prime \prime}(u)+\epsilon\left[(\sigma-1) a^{\prime}(u) d(u)+2(2-\sigma) a(u) d^{\prime}(u)\right] \frac{d(u)}{a(u)} u_{x}^{-\sigma} \\
& +\epsilon^{2}(2-\sigma)(1-\sigma) \frac{d^{3}(u)}{a(u)} u_{x}^{-2 \sigma} .
\end{aligned}
$$

Since $a(u)=(1 / m) u^{1-m}$ and $d(u)=(\alpha / m) u^{\alpha \sigma-m}$, we have

$$
\begin{array}{ll}
R(x, t)= & \frac{\alpha}{m^{2}}\{(\alpha \sigma-m)(\alpha \sigma-m-1)+\epsilon \alpha[(\sigma-1)(1-m) \\
(2.8) \quad & \left.+2(2-\sigma)(\alpha \sigma-m)] \frac{g^{\sigma}(u)}{u_{x}^{\sigma}}+\epsilon^{2} \alpha^{2}(2-\sigma)(1-\sigma) \frac{g^{2 \sigma}(u)}{u_{x}^{2 \sigma}}\right\} u^{\alpha \sigma-2 m-1} .
\end{array}
$$

Recalling (2.3) we observe that if $1-T+\tau>x_{0}$,

$$
u_{x}(x, t) \geq k g(u(x, t)) \quad \text { for }(x, t) \in[1-T+\tau, 1] \times[\tau, T)
$$

with $k$ a positive constant. Consequently, $R(x, t) \geq 0$ for small $\epsilon$, which implies

$$
\Psi_{t} \geq a(u) \Psi_{x x}+C(x, t) \Psi \quad \text { in }(1-T+\tau, 1) \times(\tau, T) .
$$

On the parabolic boundary, since $x=1$ is the only blow-up point, if $\epsilon$ is small enough, then both $\Psi(1-T+\tau, t)$ and $\Psi(x, \tau)$ are nonnegative. At $x=1$,

$$
\Psi_{x}(1, t)=b(1, t) \Psi(1, t)+\epsilon g^{2-\sigma}(u(1, t)) Q(u(1, t)),
$$

where

$$
b(x, t)=g^{\prime}(u)-\epsilon(2-\sigma) \frac{d(u)}{a(u)} g^{1-\sigma}(u)
$$


and

$$
\begin{aligned}
Q(u) & =d(u) g^{\prime}(u)-d^{\prime}(u) g(u)-\epsilon(2-\sigma) \frac{d^{2}(u)}{a(u)} g^{1-\sigma}(u) \\
& =\frac{\alpha}{m}(\alpha+m-\alpha \sigma-\epsilon \alpha(2-\sigma)) u^{\alpha \sigma+\alpha-m-1} .
\end{aligned}
$$

Since $\sigma<(m+\alpha) / \alpha$, if $\epsilon$ is sufficiently small, then $Q(u(1, t))>0$, which means that $\Psi_{x}(1, t)>b(1, t) \Psi(1, t)$ for $\tau \leq t<T$. Thus $\Psi(x, t) \geq 0$ on $[1-T+\tau, 1] \times[\tau, T)$. In particular, $\Psi(1, t) \geq 0$, that is ,

$$
u_{t}(1, t) \geq \epsilon a(u(1, t)) g(u(1, t)) g^{\prime}(u(1, t))=\frac{\epsilon \alpha}{m} u^{2 \alpha-m}(1, t) .
$$

Integration of $(2.13)$ over $(t, T)$ then leads to $(2.5)$.

\section{Blow-up RATE AND SET FOR the CASE $1<m<2 \alpha-1$}

We begin this section by presenting the lower bound on the blow-up rate for $2 \alpha>m+1$. Here we use a modification of an argument from [5].

Theorem 3.1. Let $1<m<2 \alpha-1$ and $u_{0}^{\prime}(x), u_{0}^{\prime \prime}(x) \geq 0$ on $[0,1]$. Then there exists a positive constant $C_{5}$ such that

$$
u(1, t) \geq C_{5}(T-t)^{1 /(m+1-2 \alpha)} \quad \text { for } t \in[0, T) .
$$

Proof. It suffices to prove (3.1) for $t \in[\eta, T)$ with some $\eta$ such that $u(1, \eta) \geq 1$. Set

$$
y(t)=u^{\gamma}(1, t) \int_{1-\xi(t)}^{1} u^{m}(x, t) d x
$$

with

$$
\xi(t)=(1-\epsilon) u^{1-\alpha}(1, t),
$$

where $0<\epsilon<1,(1-\epsilon) / \epsilon^{m}<2 /(\alpha-1)$, and $\alpha-m-1<\gamma \leq(\alpha-1)\left[1-m(1-\epsilon) / 2 \epsilon^{m}\right]$. A straightforward computation gives

$$
\begin{aligned}
y^{\prime}(t)= & \gamma u^{\gamma-1}(1, t) u_{t}(1, t) \int_{1-\xi(t)}^{1} u^{m}(x, t) d x \\
& +u^{\gamma}(1, t)\left(u_{x}(1, t)-u_{x}(1-\xi(t), t)\right) \\
& +(1-\epsilon)(1-\alpha) u^{\gamma-\alpha}(1, t) u^{m}(1-\xi(t), t) u_{t}(1, t) \\
\leq & -u^{\gamma-1}(1, t) u_{t}(1, t) I(t)+u^{\gamma+\alpha}(1, t),
\end{aligned}
$$

where $I(t)=(\alpha-1) u^{m}(1-\xi(t), t) \xi(t)-\gamma \int_{1-\xi(t)}^{1} u^{m}(x, t) d x$. Since $u_{x} \geq 0$ and $u_{x x} \geq 0$ in $[0,1] \times[\eta, T)$, we find

$$
\epsilon u(1, t) \leq u(1-\xi(t), t) \leq u(x, t) \leq u(1, t)
$$

for any $x \in[1-\xi(t), 1]$ and $t \in[\eta, T)$. Moreover, by (3.2), (3.3), and (3.5), we have

$$
(1-\epsilon) \epsilon^{m} u^{\gamma+m+1-\alpha}(1, t) \leq y(t) \leq(1-\epsilon) u^{\gamma+m+1-\alpha}(1, t),
$$

or equivalently,

$$
c_{1} u(1, t) \leq y^{1 /(\gamma+m+1-\alpha)}(t) \leq c_{2} u(1, t) \quad \text { for } t \in[\eta, T) .
$$


We now show that $I(t) \geq 0$ on $[\eta, T)$.

$$
\begin{aligned}
I(t)= & (1-\alpha) \int_{1-\xi(t)}^{1}\left(u^{m}(x, t)-u^{m}(1-\xi(t), t)\right) d x \\
& +(\alpha-\gamma-1) \int_{1-\xi(t)}^{1} u^{m}(x, t) d x \\
= & (1-\alpha) \int_{1-\xi(t)}^{1} m u^{m-1}(\zeta(t), t) u_{x}(\zeta(t), t)(x+\xi(t)-1) d x \\
& +(\alpha-\gamma-1) \int_{1-\xi(t)}^{1} u^{m}(x, t) d x,
\end{aligned}
$$

where $1-\xi(t)<\zeta(t)<1$. Because $\alpha, m>1, \alpha-\gamma-1>0, u_{x}, u_{x x} \geq 0$, and $\int_{1-\xi(t)}^{1}(x+\xi(t)-1) d x=(1 / 2)(1-\epsilon)^{2} u^{2-2 \alpha}(1, t)$, we can see that

$$
I(t) \geq(1-\epsilon)\left[\frac{1}{2} m(1-\alpha)(1-\epsilon)+(\alpha-\gamma-1) \epsilon^{m}\right] u^{m+1-\alpha}(1, t) \geq 0 .
$$

From (3.4), (3.7), and (3.9), it then follows that

$$
y^{\prime}(t) \leq u^{\gamma+\alpha}(1, t) \leq c_{1}^{-\gamma-\alpha} y^{(\gamma+\alpha) /(\gamma+m+1-\alpha)}(t),
$$

since $\gamma+\alpha>2 \alpha-m-1>0$. Integrating the above inequality from $t$ to $T$, we obtain

$$
y^{(m+1-2 \alpha) /(\gamma+m+1-\alpha)}(t) \leq \frac{2 \alpha-m-1}{\gamma+m+1-\alpha} c_{1}^{-\gamma-\alpha}(T-t)
$$

that is,

$$
y^{1 /(\gamma+m+1-\alpha)}(t) \geq c_{3}(T-t)^{1 /(m+1-2 \alpha)},
$$

which in conjunction with (3.7) leads to (3.1).

Based on the general idea of [7], we then locate the blow-up point for the case $(m+1) / 2<\alpha \leq m$.

Theorem 3.2. Suppose that condition $(\mathrm{H})$ holds. Then for $(m+1) / 2<\alpha \leq m$, blow-up occurs only at $x=1$.

Proof. Introduce a function $w(x) \in C^{2}([0,1])$ defined by

$$
w(x)= \begin{cases}h(x) & 0 \leq x \leq \frac{1}{2} \\ (1-x)^{2} & \frac{1}{2}<x \leq 1\end{cases}
$$

with $w^{\prime}(0)=0$ and $w^{\prime}(x) \leq 0$ for $0<x \leq 1$. Let $v(x, t)=C_{0}[w(x)+B(T-t)]^{-\rho}$, where $C_{0}$ and $B$ are positive constants to be determined, and $\rho=1 /(2 \alpha-m-1)$. Then $v(x, t)$ satisfies

$$
m u^{m-1} v_{t}-v_{x x}=C_{0} \rho[w(x)+B(T-t)]^{-\rho-1} J(x, t) \quad \text { in }(0,1) \times(0, T) .
$$

Here $J(x, t)=B m u^{m-1}-(\rho+1)\left(w^{\prime}(x)\right)^{2} /[w(x)+B(T-t)]+w^{\prime \prime}(x)$. Since $u_{0}(x) \geq$ $\delta>0$ and $u_{t} \geq 0$, for $(x, t) \in(1 / 2,1) \times(0, T)$,

$$
\begin{aligned}
J(x, t) & =B m u^{m-1}-\frac{4(\rho+1)(1-x)^{2}}{(1-x)^{2}+B(T-t)}+2 \\
& \geq B m \delta^{m-1}-4 \rho-2 \geq 0
\end{aligned}
$$


if $B \geq(4 \rho+2) / m \delta^{m-1} ;$ for $(x, t) \in(0,1 / 2] \times(0, T)$,

$$
J(x, t) \geq B m \delta^{m-1}-\frac{(\rho+1) h_{1}^{2}}{h\left(\frac{1}{2}\right)}-h_{2} \geq 0
$$

if $B \geq\left[(\rho+1) h_{1}^{2} / h(1 / 2)+h_{2}\right] / m \delta^{m-1}$, where $h_{1}=\max _{0 \leq x \leq \frac{1}{2}}\left|h^{\prime}(x)\right|$ and $h_{2}=$ $\max _{0 \leq x \leq \frac{1}{2}}\left|h^{\prime \prime}(x)\right|$.

On the parabolic boundary, $v_{x}(0, t)=u_{x}(0, t)=0$. By (1.3), if $C_{0} \geq B^{\rho} C$, then $v(1, t)=C_{0} B^{-\rho}(T-t)^{-\rho} \geq u(1, t)$; and when $t=0, C_{0}$ can be chosen so large that

$$
v(x, 0) \geq C_{0}[w(0)+B T]^{-\rho} \geq \max _{0 \leq x \leq 1} u_{0}(x) \geq u_{0}(x) .
$$

Then the maximum principle implies

$$
u(x, t) \leq v(x, t) \quad \text { for }(x, t) \in[0,1] \times[0, T),
$$

which shows that for any $x \in[0,1)$,

$$
u(x, t) \leq C_{0} w^{-\rho}(x)<\infty .
$$

Thus the proof is completed.

\section{REFERENCES}

[1] K. Deng, The blow-up behavior of the heat equation with Neumann boundary conditions, $J$. Math. Anal. Appl. 188 (1994), 641-650. MR 95i:35120

[2] K. Deng and M. Xu, On solutions of a singular diffusion equation, preprint.

[3] M. Fila and H.A. Levine, Quenching on the boundary, Nonlinear Anal. TMA 21 (1993), 795-802. MR 95b:35028

[4] M. Fila and P. Quittner, The blow-up rate for the heat equation with a non-linear boundary condition, Math. Methods Appl. Sci. 14 (1991), 197-205. MR 92a:35023

[5] J. Filo, Diffusivity versus absorption through the boundary, J. Differential Equations 99 (1992), 281-305. MR 94d:35083

[6] J.L. Gómez, V. Márquez, and N. Wolanski, Blow up results and localization of blow up points for the heat equation with a nonlinear boundary condition, J. Differential Equations 92 (1991), 384-401. MR 92j:35098

[7] B. Hu and H.-M. Yin, The profile near blow-up time for solutions of the heat equation with a nonlinear boundary condition, Trans. Amer. Math. Soc. 346 (1994), 117-135. MR 95c:35040

[8] N. Wolanski, Global behavior of positive solutions to nonlinear diffusion problems with nonlinear absorption through the boundary, SIAM J. Math. Anal. 24 (1993), 317-326. MR 93j:35023

Department of Mathematics, University of Southwestern Louisiana, Lafayette, LOUISIANA 70504

E-mail address: kxd5858@usl.edu

E-mail address: mxx3473@usl.edu 